\title{
Oxygenic photosynthesis and the distribution of chloroplasts
}

\author{
Elisabeth Gantt
}

Published online: 29 December 2010

(C) Springer Science+Business Media B.V. 2010

\begin{abstract}
The integrated functioning of two photosystems (I and II) whether in cyanobacteria or in chloroplasts is the outstanding sign of a common ancestral origin. Many variations on the basic theme are currently evident in oxygenic photosynthetic organisms whether they are prokaryotes, unicellular, or multicellular. By conservative estimates, oxygenic photosynthesis has been around for at least ca. 2.2-2.7 billions years, consistent with cyanobacteria-type microfossils, biomarkers, and an atmospheric rise in oxygen to less than $1.0 \%$ of the present concentration. The presumptions of chloroplast formation by the cyanobacterial uptake into a eukaryote prior to $1.6 \mathrm{BYa}$ ago are confounded by assumptions of host type(s) and potential tolerance of oxygen toxicity. The attempted dating and interrelationships of particular chloroplasts in various plant or animal lineages has relied heavily on phylogenomic analysis and evaluations that have been difficult to confirm separately. Many variations occur in algal groups, involving the type and number of accessory pigments, and the number(s) of membranes (2-4) enclosing a chloroplast, which can both help and complicate inferences made about early or late origins of chloroplasts. Integration of updated phylogenomics with physiological and cytological observations remains a special challenge, but could lead to more accurate assumptions of initial and extant endosymbiotic event(s) leading toward stable chloroplast associations.
\end{abstract}

Keywords Chloroplast types - Cyanobacteria . Eukaryotic algae $\cdot$ Nucleomorph $\cdot$ Reaction centers

E. Gantt $(\square)$

Department of Cell Biology and Molecular Genetics, University of Maryland, College Park, MD 20742, USA

e-mail: egantt@umd.edu

\author{
Abbreviations \\ BYa Billion years ago \\ Chl Chlorophyll \\ EGT Endosymbiotic gene transfer \\ HGT Horizontal (lateral) gene transfer \\ OEC Oxygen evolving complex \\ PAL Present atmospheric level (of oxygen) \\ PSI, PSII Photosystem I, Photosystem II, respectively
}

\section{Introduction}

Present day life as we know it is dependent on oxygenic photosynthesis. It provides breathable air, and photosystem II can derive an unlimited source of electrons from water by using energy from the sun. The co-editors of this volume (Gantt and Falkowski) have invited specialists from a broad range of disciplines to benefit those readers interested in a comprehensive understanding of oxygenic photosynthesis. Major topics being addressed in the accompanying series of articles relate to the evidence and time-lines of oxygenic photosynthesis on the earth (Farquhar et al. 2010), the resultant gains of an aerobic atmosphere and the increase in organismal size and diversity, as well as multicellularity (Payne et al. 2010). At the organismal level, some of the biggest questions are: what were the original key characteristics from which the photosynthetic reaction centers were derived (Allen and Williams 2010), what essential changes were required for electron production by the water splitting complex (Williamson et al. 2010), and what is the evidence for the timeline of how long cyanobacteria have been around (Schopf 2010)? Present day chloroplasts, presumably all derived originally from one cyanobacterial endosymbiotic event, have become dispersed in single-celled eukaryotic 
"hosts" with the greatest dispersion among the chlorophyll $c$-containing algae (Green 2010). Numerous examples of symbiotic stages of photosynthetic organisms in multicellular animals (Johnson 2010) lead to the interesting possibility that many of these are present day examples of chloroplast evolution in action, i.e., possible progressions from the symbiotic toward the endosymbiotic state.

The contributing authors are specialists in their respective areas with different approaches, with all of them providing valuable critical views and updates of their fields. Their contributions with their own interpretations and evaluations is what makes this a combined richer offering, especially since all the areas covered continue to be actively explored, and hence change as new methods lead to new data and often to new interpretations. This author may be faulted for questioning some of the long held simple assumptions of chloroplast origins, especially the presumption of a relatively simple progression from one endosymbiotic event. In no way should this be interpreted as a criticism of past interpretations from limited data, but perhaps it may serve as impetus toward the re-examination of some embedded paradigms.

\section{Correlating rise of oxygenic atmosphere with the presence of cyanobacteria}

Cyanobacteria are almost universally regarded as the initial providers of oxygen to the oceans and atmosphere, but hypotheses have varied as to when cyanobacteria first arose. This group may date to Archean times (ca. 3.5 BYa) when anoxygenic conditions prevailed. Among geologists and geochemists, it is generally agreed that the atmosphere and oceans were devoid of oxygen until ca. 2.45 BYa, the time of the great oxidation event (Canfield 2005; Farquhar et al. 2010). Yet considerable allowances have to be made for a lag in time, differences in local environments before the notable $\mathrm{O}_{2}$ rise resulted in a transition from anoxia to the estimated ca. $0.001-1.0 \% \mathrm{O}_{2}$ concentration of present (PAL) (Payne et al. 2010).

When and how cyanobacteria arose has been difficult to establish. Previously, morphological size and shape were the main criteria by which cyanobacterial-type fossils were identified. Because of complications arising from the destruction of fossil features by pressure, heat, and chemical alterations over time, differences in interpretations have sometimes greatly differed when morphology alone was used. One of the oldest (3.45 BYa) fossils with biogenic traces and organismal morphologies are found in the Strelley Pool Chert from the Pilbara Craton in Australia (Allwood et al. 2009). Rich sources of cyanobacterial-like microfossils occur in stromatolites (laminated structures of carbonate or silicate rocks) from many other regions of the world and various continents (e.g., Schopf 2010). However, some of the oldest microfossils have been evaluated differently, either as simple non-organismal accretions (Brasier et al. 2002) or as impressions of cyanobacterialtype cells (Schopf et al. 2002). As detailed in the chapter by Schopf (2010), additional analytical methods have greatly increased the confidence in both dating and identification of the cyanobacterial-type microfossils of stromatolites from many geographical regions. The combined results leave little doubt that cyanobacterial-type organisms existed well prior to $2.5 \mathrm{BYa}$, i.e., long before a significant rise in atmospheric oxygen.

\section{Two photosystems and the water splitting complex}

The deposition of sedimentary organic matter also can also be correlated with changes in the nitrogen cycle (Farquhar et al. 2010 and references therein) that would likely have involved the cyanobacteria as significant contributors. However, the general assumption is that much of the fixed carbon in sediments is from anoxic photosynthetic bacteria, the presumed independent progenitors of the photosynthetic reaction centers (RCI, RCII). It has been argued convincingly that extant photosynthetic bacteria (green sulfur bacteria and acidobacteria) are the precursors for photosystem I (RCI). Similarly, there are strong structural similarities of green filamentous bacteria and purple bacteria (Bryant and Frigaard 2006) that are persuasive as potential progenitors of the extant photosystem II. The elucidation of the crystal structure of the RC from purple bacteria (Deisenhofer et al. 1985) made it clear that the core components of the PSII reaction center (RCII) are very similar. However, the bacterial reaction centers cannot oxidize water despite the similarity of protein structures and likely evolutionary relationship to photosystem II (Sadekar et al. 2006; Allen and Williams 2010 and references therein).

There are some major issues that do not support (Green and Gantt 2000) assumptions that the RCs were gained from photosynthetic bacteria: the bacterial chlorophylls have considerably longer wavelength absorptions, evidence is lacking as to how the bacterial reaction centers could have combined, it is not apparent what might have lead to the altered the photosynthetic pigmentation, and especially the negative effects attendant from aerobic photosynthesis. It appears to be more logical to assume that extant photosynthetic bacteria adapted specifically to their current ecological niches, rather than to assume that they have been preserved in their present form since Archean times. Certainly functional similarities occur between reaction center types, but this probably tells us very little at this point about their respective ancestral origins. 
The predominant photosynthetic pigment absorption ranging from cyanobacteria to trees, is in the visible light spectrum (ca. 400-700 nm). This could reflect functional adaptations that maximized their success, i.e., the development of oxygenic organisms. Chlorophyll $a$ is always the central chlorophyll in oxygenic plants. Interestingly, many other pigment types fill an optical gap (ca. 445-670 nm) (Jeffrey et al. 1997) where Chl $a$ absorption is minimal. Such accessory pigments are synthesized by a variety of divergent biosynthetic pathways. Major accessory pigments include $\mathrm{Chl} b$, Chl $c$, the phycobiliproteins, and the carotenoid-based fucoxanthins and peridinin. Rarely do extant oxygenic organisms possess chlorophylls with a longer wavelength range to ca. $720 \mathrm{~nm}$, e.g., Chl $d$ (Allakhverdiev et al. 2010) and even $\mathrm{Chl} f$ (Chen et al. 2010). Are these rare chlorophylls to be regarded as evolutionary remnants, as evolutionary transitions, or as interesting variants that do not represent direct clues to or from a major evolutionary path? The latter option seems the most rational at this time.

The primary distinction and most unifying feature in the evolutionary development of oxygenic photosynthesis is also the most confounding puzzle. When and how did the water splitting complex (OEC) develop and become integrated into photosystem II, with the concomitant cooperation of photosystem I? It could have originated from a di-iron complex wherein the Fe was substituted by $\mathrm{Mn}$ thereby forming a Mn-catalase; however, there is a lack of protein sequence homology with the OEC (Raymond and Blankenship 2008). A number of additional interesting suggestions on the potential origin of the key features are reviewed by Williamson et al. (2010 and references therein).

\section{Puzzling on chloroplast ancestry from an initial endosymbiotic event}

It is widely accepted that chloroplasts are derived from a single one-time event where a cyanobacterium was taken up into a eukaryotic single-celled organism (Delwiche 1999) which formed the base for all eukaryotic photosynthetic organisms (Green 2010; Ryes-Prieto et al. 2008; Yoon et al. 2004). This idea has become a paradigm that is widely illustrated in text books and continues to have considerable support from phylogenomic analyses (Hackett et al. 2007; Keeling 2010). Phylogenetic analyses indeed can be constructed to show that extant cyanobacteria fall into a monophyletic line and suggest that the heterocyst formers diverged when atmospheric $\mathrm{O}_{2}$ concentrations increased (Tomitani et al. 2006) around the time of the great oxidation event. The reductive reasoning of a onetime uptake of a cyanobacterium, into one eukaryotic host, followed by linear descent of photosynthetic eukaryotes, although logically appealing appears to be countered by widely observed biological diversity.

One critical assumption is that the eukaryotic host cell for the cyanobacterium already contained a mitochondrion derived from an $\alpha$-proteobacterial ancestor (Gray et al. 2001). This raises the question of whether, and if, the mitochondrial progenitor and its eukaryotic host were already tolerant of the toxic effects (Aple and Hirt 2004) from $\mathrm{O}_{2}$ which would have been generated by the cyanobacterial endosymbiont's photosynthesis. Thus, it has to be assumed that (1) the mitochondrial-bacterial-progenitor had evolved in an oxygenic environment or that (2) a rapid tolerance to oxygenic damaging effects developed after entry of the oxygen producing cyanobacterial endosymbiont with extant characteristics. A scenario of gradual adaptation toward oxygen production in transition forms, and the subsequent acquisition of a proteobacterial-like mitochondrial ancestor would be more biologically logical.

Best estimates suggest that the concentration of $\mathrm{O}_{2}$ was still rather low (Fig. 1, Payne et al. 2010; Frei et al. 2009) at the time when the proposed cyanobacterial-to-chloroplast uptake occurred in the early Proterozoic Eon. A potential eukaryotic host could have come from the base of the animal ancestral lineage, possibly related to opisthokonts (Yoon et al. 2004). According to timeline calculations by Yoon et al. (2004), the cyanobacterial endosymbiotic event of the cyanobacterial-to-chloroplast transition would have been somewhat prior to ca. 1.6 BYa with a subsequent split into the glaucophyte and red-green algal separation. Current extant opisthokonts are aquatic single-celled heterotrophs usually with a single flagellum, which feed on detritus including bacteria and phytoplankton. If a flagellated organism was indeed an early eukaryotic host, it must have been very different from the extant flagellated forms that require a highly aerobic environment.

\section{Distribution of chloroplasts: finding Cinderella's slipper}

Three chloroplast lineages (glaucophyte, red, and green) are presumed to have arisen from a single primary endosymbiosis of a cyanobacterium into a eukaryotic host, from which they descended as a monophyletic lineage. Whether or not the three groups are viewed as monophyletic or polyphyletic, and which is placed at the base of the "clade," depends on interpretation of divergent evidence and the assignation of importance to various selected gene sets. In spite of numerous publications, the debate continues (cf. in Green 2010; Baurian et al. 2010; Deschamps and Moreira 2009; Janouškovec et al. 2010; Keeling 2010; Nozaki et al. 2009; Ryes-Prieto et al. 2008; Stiller 2007). Many attempts have focused on trying to ascertain if there 
was one chloroplast origin, and if so, what was the most likely host, i.e., is there only one Cinderella slipper and where is the best fit?

Some unambiguous structural signs of symbiotic and/or endosymbiotic events were found some years ago when Gibbs (1981) provided significant examples showing that some chloroplasts had two limiting membranes (green and red algae), others were surrounded by three membranes (euglenids, dinoflagellates), while still others had four chloroplast membranes (browns, diatoms, cryptophytes) usually with an additional set of ribosomes on the "chloroplast endoplasmic reticulum." Cryptophytes even contained a remnant of a nucleus (nucleomorph) albeit with a small genome but with some 30 chloroplast genes along with housekeeping genes to permit their expression (reviewed by Archibald 2007).

\section{Glaucophyte lineage}

The blue-green cyanobacterial-type inclusions are justified as being functional chloroplasts (organelles) in the glaucophytes. Because they have remnants of a peptidoglycan layer, plus carboxysome-type bodies, they have been regarded as transitional forms of plastids (Cavalier-Smith 2002; Steiner and Loeffelhardt 2002; Deschamps and Moreira 2009); however, the host ancestry is poorly explored and usually has not factored heavily into lineage considerations. For instance, the identifying species Glaucocystis nostochinearum is a non-motile unicell with a cellulosic wall, while Cyanophora paradoxa is a bi-flagellated motile unicell. On the other hand, Paulinella chromatophora is an ameba with cyanobacterial inclusions, but it is not included in the chloroplast lineage (Bodyl et al. 2010). Various indicators are that the cyanobacterial-type inclusions are transition states; but did they become developmentally stuck for possibly 1.6 BYa in their progression toward the chloroplast state, or are all these association much more recent? It seems highly unlikely that they would have been stuck in their state for eons, and it is more likely that they are relatively recent transition states.

\section{Green algal linage}

With Chl $a$ and $b$ as typifying pigments, two chloroplastlimiting membranes, and granal-stacked thylakoids, there is little discussion of the accepted monophyly of chloroplasts from flagellated unicellular algae to vascular plants. The common assumption is that the cyanobacterial ancestor lost the phycobiliproteins as accessory pigments and substituted Chl $b$ and certain carotenoids to enhance the absorption capacity. The chloroplasts of the green lineage appear to be rather stable biochemically and structurally. The possibility that $\mathrm{Chl} a / b$ containing prokaryotes might be regarded as potential progenitors of green plants has not gained much support (La Roche et al. 1996).

Other groups, with $\mathrm{Chl} a$ and $b$ pigmentation, are euglenids and chlorarachniophytes for which two separate secondary endosymbioses have been suggested (Green 2010, Fig. 1) but with distinctly different hosts. One example is Euglena, a flagellate with three membranes surrounding its chloroplast. A different example is Bigelowiella, which has four membranes surrounding the chloroplasts, has a nucleomorph (Archibald 2007), but is encased in an ameba.

\section{Red algal lineage}

The red algal group appears to be another stable chloroplast lineage with two chloroplast-limiting membranes and a simple photosynthetic pigment combination of Chl $a$ and phycobiliproteins, a pigmentation virtually identical to that of cyanobacteria. Also, this lineage has one of the oldest and structurally most convincing fossil remnants at ca. 1.2 BYa (Butterfield 2000). Nevertheless, the group has been at the center of the chloroplast dispersion controversy mostly because it has been placed as endosymbiont at the base of the chromalveolates, argued to be a monophyletic evolutionary group (Cavalier-Smith 2002; cf. Green 2010; Janouškovec et al. 2010). The chromalveolates are a diverse grouping distinguished by: the presence of $\mathrm{Chl} a$ plus $\mathrm{Chl} c$, carotenoid-type fucoxanthin or peridinin, having ciliated or flagellated hosts, and by some un-pigmented members having presumably lost a once functioning integrated chloroplast. Significant aspects of the chromalveolate hypothesis and major questions are provided by Green (2010) in a critical synopsis. She points out some of the unresolved problems, such as trying to reconcile the wide diversity of hosts with a single red algal endosymbiosis and the positioning of un-pigmented species.

An important postulation for coherence of the chromalveolates as a natural group is an explanation accounting for the presence of fully heterotrophic members that lack a plastid. A seemingly logical explanation has been to postulate a significant reduction of chloroplast-related genes or an outright loss (Cavalier-Smith 2002). Resolving questions of gene reduction, and whether specific individual genes were retained after a plastid loss, has been difficult to substantiate partly due to an unequal weighting of selected gene comparisons and because of some presumed chloroplast genes which have broader cell functions. Cautions against oversimplifications from gene sampling and potential losses are valid and are growing (Archibald 2009; Bodyl et al. 2009; Howe et al. 2008; Inagaki et al. 2009; Stiller 2007; Stiller et al. 2009), though not always popular with the current multitude who continue to try to find the right place(s) for Cinderella's slipper. 
Transitory and constant associations

There are multiple extant states of symbiotic associations between aquatic animals and photosynthetic organisms, both at the single cell level and multicellular levels. Many of them provide clues that might be useful in alternate considerations of how plastids differentiated and spread. An elaboration of many such examples is illustrated in the chapter by Johnson (2010) dealing with adaptive strategies in hosting cells and their organelles. These adaptive strategies are mutualistic and are generally driven by the sharing of basic metabolic resources. Dinoflagellate associations with coral tissue appear to be rather common, as are hydra and green algal associations (Trench 1979). To what extent there has been gene transfer between host and symbiont is generally not known. However, gene transfers between two very different $\mathrm{Chl} a / b$ algae to sea slug hosts have been demonstrated (Rumpho et al. 2008; Pierce et al. 2007). Another example from Stoecker's laboratory (Johnson et al. 2007) highlights a ciliate that "fed" on flagellated cryptophytes and retained transcriptionally active cryptophyte nuclei. Such examples clearly suggest that transfer of genetic content is not uncommon among algal groups and hosts. Yet, these associations, whether transitory or relatively stable, do not necessarily lead to evolutionary progressions as has been so commonly inferred for chloroplast lineage(s), especially among the collection of algae placed in the chromalveolate group.

\section{Summary opinion}

The assumption of a one-time chloroplast origin and subsequent dispersals via specific hosts is clearly under threat from new data and multiple interpretations. With ever increasing examples of gene transfers (HGT, EGT) among prokaryotes and eukaryotes, of fungi to animals (Moran and Jarvick 2010), and between algae and animals, it is difficult to cling to some of the presently strongly held concepts of strictly linear progressions on which widely accepted models for the evolution of photosynthesis are based. It seems very unlikely that there was a straight linear progression to a PSI-PSII progenitor and one endosymbiotic cyanobacteria to chloroplast occurrence.

Many phylogenomic applications have narrowed, rather than broadened, our views of evolutionary progressions. When considered from a broader biological perspective, without historically outdated assumptions often made from analysis of few selected genes, it is clear that there is considerable "evolution in action" at the present time. However, many of the naturally occurring associations are probably transient and are unlikely to be on an advancing tract toward stable long-term endosymbioses and/or fully integrated plastids. Sorting out which groups are more stable, and which individuals and/or groups are in the process of adapting to environmental conditions, are challenges for which the present concepts have become inadequate.

Acknowledgments With special thanks for the input by JWS, BRG, and RRG.

\section{References}

Allakhverdiev SI, Tomo T, Shimada Y, Kindo H, Nagao R, Klimov VV, Mimuro M (2010) Redox potential of pheophytin a in photosystem II of two cyanobacteria having the different special pair chlorophylls. PNAS 107:3924-39249

Allen JP, Williams JC (2010) The evolutionary pathway from anoxygenic to oxygenic photosynthesis examined by comparison of the properties of photosystem II and bacterial reaction centers. Photosynth Res. doi:10.1007/s11120-010-9552-x

Allwood AC, Grotzinger JP, Knoll AH, Burch IW, Anderson MS, Coleman ML, Kanik I (2009) Controls on development and diversity of Early Archean stromatolites. PNAS 106:9548-9555

Aple K, Hirt H (2004) Reactive oxygen species: metabolism, oxidative stress, and signal transduction. Annu Rev Plant Biol 55:373-399

Archibald JM (2007) Nucleomorph genomes: structure, function, origin and evolution. BioEssays 29:392-402

Archibald JM (2009) The puzzle of plastid evolution. Curr Biol 19:RS81-RS88

Baurian D, Brinkmann H, Petersen J, Rodriguez-Ezpeleta N, Stechmann A, Demoulin V, Roger AJ, Burger F, Lang BF, Philippe H (2010) Phylogenomic evidence for separate acquisition of plastids in cryptophytes, haptophytes, and stramenopiles. Mol Biol Evol 27:1698-1709

Bodyl A, Mackiewicz P, Stiller JW (2009) Early steps in plastid evolution: current ideas and controversies. BioEssays 31: $1219-1232$

Bodyl A, Mackiewicz P, Stiller JW (2010) Comparative genomic studies suggest that the cyanobacterial endosymbionts of the amoeba Paulinella chromatophora possess an import apparatus for nuclear-encoded proteins. Plant Biol (Stuttg) 12:639-649

Brasier MD, Green OR, Jephcoat AP, Kleppe AK, Van Kranendonk MJ, Lindsay JF, Steele A, Grassineau NV (2002) Questioning the evidence for Earth's oldest fossils. Nature 416:76-81

Bryant D, Frigaard N-U (2006) Prokaryotic photosynthesis and phototrophy illuminated. Trends Microbiol 14:488-496

Butterfield NJ (2000) Bangiomorpha pubescens n. gen., n. sp.: implications for the evolution of sex, multicellularity, and the Mesoproterozoic/Neoproterozoic radiation of eukaryotes. Paleobiology 26:386-404

Canfield DE (2005) The early history of atmospheric oxygen: homage to Robert M. Garrels. Annu Rev Earth Planet Sci 33:1-36

Cavalier-Smith T (2002) Chloroplast evolution: secondary symbiogenesis and multiple losses. Curr Biol 12:R62-R64

Chen M, Schliep M, Willows RD, Cai Z-L, Neilan BA, Scheer H (2010) A red-shifted chlorophyll. Science 329:1138-1319

Deisenhofer J, Epp O, Miki K, Huber R, Michel H (1985) Structure of the protein subunits in the photosynthetic reaction centre of Rhodopseudomonas viridis at 3 A resolution. Nature 318:618-624

Delwiche CF (1999) Tracing the thread of plastic diversity through the tapestry of life. Am Nat 154:164-177

Deschamps P, Moreira D (2009) Signal conflicts in the phylogeny of the primary photosynthetic eukaryotes. Mol Biol Evol 26: $2745-2753$ 
Farquhar J, Zerkle AL, Bekker A (2010) Geological constraints on the origin of oxygenic photosynthesis. Photosynth Res. doi:10.1007/ s11120-010-9594-0

Frei R, Gaucher C, Poulton SW, Canfield DE (2009) Fluctuation in Precambrian atmospheric oxygenation recorded by chromium isotopes. Nature 461:250-254

Gibbs SP (1981) The chloroplast endoplasmic reticulum: structure, function, and evolutionary significance. Int Rev Cytol 72:49-99

Gray JW, Burger G, Lang BF (2001) The origin and early evolution of mitochondria. Genome Biol 2:reviews1018.1-1018.5

Green BR (2010) After the primary endosymbiosis: an update on the chromalveolate hypothesis and the origins of algae with $\mathrm{Chl} \mathrm{c.}$ Photosynth Res. doi:10.1007/s11120-010-9584-2

Green BR, Gantt E (2000) Is photosynthesis really derived from purple bacteria? J Phycol 36:983-985

Hackett JD, Yoon HS, Li S, Reyes-Prieto A, Rummele SE, Bhattacharya D (2007) Phylogenomic analysis support the monophyly of cryptophytes and haptophytes and the association of rhizaria with chromalveolates. Mol Biol Evol 24:1702-1713

Howe CJ, Barbrook AC, Nisbet RER, Lockhart PJ, Larkum (2008) The origin of plastids. Philos Trans R Soc B 363:2675-2685

Inagaki Y, Nakajima Y, Sato M, Sakaguchi M, Hashimoto T (2009) Gene sampling can bias multi-gene phylogenetic inferences: the relationship between red algae and green plants as a case study. Mol Biol Evol 26:1171-1179

Janouškovec J, Horák A, Obornik M, Luke J, Keeling PJ (2010) A common red algal origin of the apicomplexan, dinoflagellate, and heterokont plastids. PNAS 107:10949-10954

Jeffrey SW, Mantoura RFC, Wright SW (1997) Phytoplankton pigments in oceanography: guidelines to modern methods. UNESCO Publishing, France

Johnson MD (2010) The acquisition of phototrophy: adaptive strategies of hosting endosymbionts and organelles. Photosynth Res. doi:10.1007/s11120-010-9546-8

Johnson MD, Oldach D, Delwiche C, Stoecker DK (2007) Retention of transcriptionally active cryptophyte nuclei by the ciliate Myrionecta rubra. Nature 445:426-428

Keeling PJ (2010) The endosymbiotic origin, diversification and fate of plastids. Philos Trans R Soc Lond B Biol Sci 365:729-748

La Roche J, van der Staay GWM, Partensky F, Ducret A, Aebersold R, Li R, Golden SS, Hiller RG, Wrench PM, Larkum AWD, Green BR (1996) Independent evolution of the prochlorophyte and green plant chlorophyll $a / b$ light-harvesting proteins. PNAS 93:15244-15248

Moran NA, Jarvick T (2010) Lateral transfer of genes from fungi underlies carotenoid production in aphids. Science 328:624-627

Nozaki H, Maruyama M, Matsuzaki M, Nakada T, Kato S, Misawa K (2009) Phylogenetic positions of Glaucophyta, green plants
(Archaeplastida) and Haptophyta (Chromalveolata) as deduced from slowly evolving nuclear genes. Mol Phylogenet Evol 53:872-880

Payne JL, McClaim CR, Boyers AG, Brown JH, Finnegan S, Kowalewski M, Krause RA, Lyosn SK, McSheas DW, NovackGottshall PM, Smith FA, Spaeth P, Stempient J, Wang SC (2010) The evolutionary consequences of oxygenic photosynthesis: a body size perspective. Photosynth Res. doi: 10.1007/s11120-010-9593-1

Pierce SK, Curtis NE, Hanten JJ, Boerner SL, Schwartz JA (2007) Transfer, integration and expression of functional nuclear genes between multicellular species. Symbiosis 43:57-64

Raymond J, Blankenship RE (2008) The origin of the oxygenevolving complex. Coord Chem Rev 252:377-383

Rumpho ME, Worful JM, Lee J, Kannan K, Tyler MS, Bhattacharya D, Moustafa A, Manhart JR (2008) Horizontal gene transfer of the algal nuclear gene psbO to the photosynthetic sea slug Elysia chlorotica. PNAS 105:17867-17871

Ryes-Prieto A, Moustafa A, Bhattacharya D (2008) Multiple genes of apparent algal origin suggest ciliates may once have been photosynthetic. Curr Biol 18:956-962

Sadekar S, Raymond J, Blankenship RE (2006) Conservation of distantly related membrane proteins: photosynthetic reaction centers share a common structural core. Mol Biol Evol 23: 2001-2006

Schopf JW (2010) The paleobiologic record of photosynthesis. Photosynth Res. doi:10.1007/s11120-010-9577-1

Schopf JW, Kudryavtsev AB, Agresti DG, Wdowiak TJ, Czaja AD (2002) Laser-Raman imagery of Earth's earliest fossils. Nature 416:73-76

Steiner JM, Loeffelhardt W (2002) Protein import into cyanelles. Trends Plant Sci 7:72-77

Stiller JW (2007) Plastid endosymbiosis, genome evolution and the origin of green plants. Trends Plant Sci 12:391-396

Stiller J, Huang WJ, Ding Q, Tian J, Goodwillie C (2009) Are algal genes in nonphotosynthetic protists evidence of historical plastid endosymbioses? BMC Genomics 10:484

Tomitani A, Knoll AH, Cavanaugh CM, Ohno T (2006) The evolutionary diversification of cyanobacteria: molecular-phylogenetic and paleontological perspectives. PNAS 103:5442-5447

Trench RK (1979) Cell biology of plant-animal symbiosis. Annu Rev Plant Physiol Plant Mol Biol 30:485-531

Williamson A, Conlan B, Hillier W, Wydrzynski T (2010) The evolution of photosystem II-insights into the past and future. Photosynth Res. doi:10.1007/s11120-010-9559-3

Yoon HS, Hackett JD, Ciniglia C, Pinto G, Bhattacharya (2004) A molecular timeline for the origin of photosynthetic eukaryotes. Mol Biol Evol 21:809-818 